\title{
Changes in motor unit populations in motor neurone disease
}

\author{
S. A. CARLET O N A N W F F B O W N \\ From the University Hospital, London, Ontario, Canada
}

SUMMARY In motor neurone disease changes in the functional properties of motor units, including the surface voltage, latency, conduction velocity, and response to repetitive stimulation, were investigated. Progression was marked by motor unit loss, increase in the proportion of larger motor unit potentials, and inclusion of motor unit potentials larger than normal in the remaining motor unit population. Even late in the disease, motor unit potentials with a low surface voltage persisted. The relationship between motor unit potentials, surface voltage, and latency, present in control subjects, broke down in motor neurone disease, large motor unit potentials having abnormally long latencies and small motor unit potentials unexpectedly short latencies. Amplitude decrements were more frequent and severe in motor unit potentials at later stages in the disease, particularly in those units with lower surface voltages. In one surviving motor unit potential there was evidence suggestive of functional recovery. The observations point to complex changes in the functional properties of motor units in motor neurone disease.

In myasthenia gravis, neuromuscular transmission abnormalities were most frequent and severe in hand motor unit potentials (MUPs) with the lowest voltages measured on the skin surface over the muscle(s) tested (Kadrie and Brown, 1978b). In the uncommon peripheral neuropathies with abnormal neuromuscular transmission, and much more commonly in motor neurone disease (MND), this observation was repeated. The fact that the above observation was present in three unrelated disorders prompted a new hypothesis.

Could the margin for safe neuromuscular transmission, even in health, be less for those motor units with MUPs of lower rather than higher surface voltages? If true, failures in neuromuscular transmission might be expected to occur earliest, more commonly, and in a more severe form at neuromuscular junctions belonging to those motor units that had the lowest voltage potentials measured over the muscle surface. This hypothesis and other questions lead us to present for critical review, our observations from MND patients on the behaviour of MUPs of low to high surface voltage in tests of neuromuscular transmission.

Address for reprint requests: Dr W. F. Brown, Department of Clinical Neurological Sciences, University Hospital, PO Box 5339, London, Ontario, Canada N6A 5A5.

Accepted 10 July 1978
Abnormal decrements in MUP voltage, evoked by trains of stimuli have been reported frequently in MND (Simpson, 1966; Lambert, 1969; Brown and Jaatoul, 1974; Norris, 1975). Nothing has been learned, however, about whether the relative frequency or severity of abnormalities in neuromuscular transmission was related to the latencies or surface voltages of the MUPs tested. We hoped, moreover, to learn from comparison of the surface voltage distributions, latencies, and responses to neuromuscular transmission tests, what changes took place in the functional properties of motor units that parallel the severity and stage of the MND involvement of the test muscles. To look at the above question, the Multiple Point Stimulation method (Kadrie et al., 1976) was chosen, permitting the isolation and investigation of 2-20 hypothenar or thenar MUPs per patient including the chance to test neuromuscular transmission at the level of the individual motor unit.

\section{Methods}

For this investigation, 13 patients with motor neurone disease, all less than 60 years of age, were tested (Table 1). Patients over the age of 60 years were excluded because of previous evidence of significant motor unit loss over that age (Brown, 
1972; Campbell et al., 1973; McComas, 1977). Disease duration at the time of initial testing ranged from 0.5 to 12 years. Disease duration is important because in MND, $50 \%$ of patients live for less than three years, up to $20 \%$ living longer than 20 years (Mulder and Howard, 1976). In this investigation close to $50 \%$ of patients had lived longer than three years from the onset of complaints.

Disease duration, however, may not reflect the rate of progression or state of involvement of a muscle being tested. Therefore, as an index of the total number of innervated muscle fibres, though not the number of motor units, the hypothenar or thenar maximum compound potential (MCP) surface peak-to-peak voltage ( $\mathrm{p}-\mathrm{pV})$ was chosen. The control MCP two standard deviation (SD) lower limit was used to divide patients into two groups: group 1 represented earlier less involved and group 2 later more advanced stages, all muscles from the latter having obvious muscle wasting.

The hypothenar and thenar muscle groups and the respective motor nerves were tested using methods previously reported (Kadrie et al., 1976; Kadrie and Brown, 1978a). In control subjects there is a significant correlation between motor unit tension and the voltage recorded on the surface of the muscle for the first dorsal interosseous (Milner-Brown and Stein, 1975), hypothenar (Kadrie et al., 1976), and the thenar muscles (Brown, unpublished), MUPs with higher surface voltages being associated with larger tensions than lower surface voltage MUPs. There is also a significant relation between motor unit tension and latency (Freund et al., 1975). Because of the above evidence the surface voltage and latency of MUPs were measured for each motor fibre excited at or just above motor threshold by means of the Multiple Point Stimulation method (Kadrie et al., 1976). The fact that the motor fibres were not all stimulated at equal distances from the motor point required the latencies to be adjusted to a standard distance from the motor point in order to facilitate comparison of latencies from different MUPs. The surface electrode types and arrangements have been described previously (Brown and Jaatoul, 1974; Kadrie et al., 1976).

Conventional methods were used to measure the maximum motor conduction velocity (MCV) and terminal motor latencies. The residual latencies were measured by methods previously described (Kadrie and Brown, 1978a).

Neuromuscular transmission was tested by measuring the changes in the $\mathrm{p}-\mathrm{pV}$ and peak-topeak duration $(\mathrm{p}-\mathrm{pD})$ of the individual hypothenar or thenar MUPs and the respective maximum com- pound potentials evoked by nerve stimulation at different stimulus intervals using methods reported in detail elsewhere (Kadrie and Brown, 1978a).

Motor unit estimates (MUEs) from the hypothenar or thenar muscles were made by methods that corrected for overlap in the critical thresholds of the first two to five motor nerve fibres excited above motor thresholds (Milner-Brown and Brown, 1976).

The motor conduction velocities of single MUPs could be measured directly in cases where only single MUPs remained.

\section{Results}

The observations from all MND patients tested are summarised in Table 1 . The maximum conduction velocities from the ulnar and median nerve and the ulnar residual latency measurements were in the normal range, and there was no significant difference between MND and control subjects. The residual latencies in the median nerve from MND patients were, however, significantly longer compared to controls.

\section{RELATIONSHIP OF THE MAXIMUM COMPOUND} POTENTIAL IN MND TO THE MOTOR UNIT ESTIMATE On the basis of the initial test results, 10 out of 15 hypothenar and five out of six thenar maximum compound potentials (Fig. 1) were less than the two standard deviation lower limit for controls and were, therefore, classified in group 2. In group 2, motor unit loss was severe, all motor unit estimates being less than 30 . Muscle atrophy was severe in nine and moderate in five of the 16 muscle groups tested in this group.

In group 1, however, more motor units were present (range 43-225, mean 120 \pm 70 ), and muscle atrophy was less frequent and severe; being just noticeable in half and not evident at all in one third of the patients. It was obvious that, at least for some subjects, muscle bulk and the maximum compound potential could be maintained at or close to the normal range despite large losses of motor units. At late stages, however, when motor unit loss was much more advanced (motor unit estimates less than 30), muscle wasting, usually severe, was invariably present.

\section{DISTRIBUTION OF SURFACE VOLTAGES OF MOTOR} UNIT POTENTIALS IN MND

Motor unit potentials isolated by means of threshold or near threshold nerve stimulation have been classified into small $(<0.049 \mathrm{mV})$, medium $(0.05-0.149 \mathrm{mV})$, and large $(>0.150 \mathrm{mV})$, the ranges being arbitrary divisions chosen to facilitate the 
Table 1 Summary of observations on patients with motor neurone disease

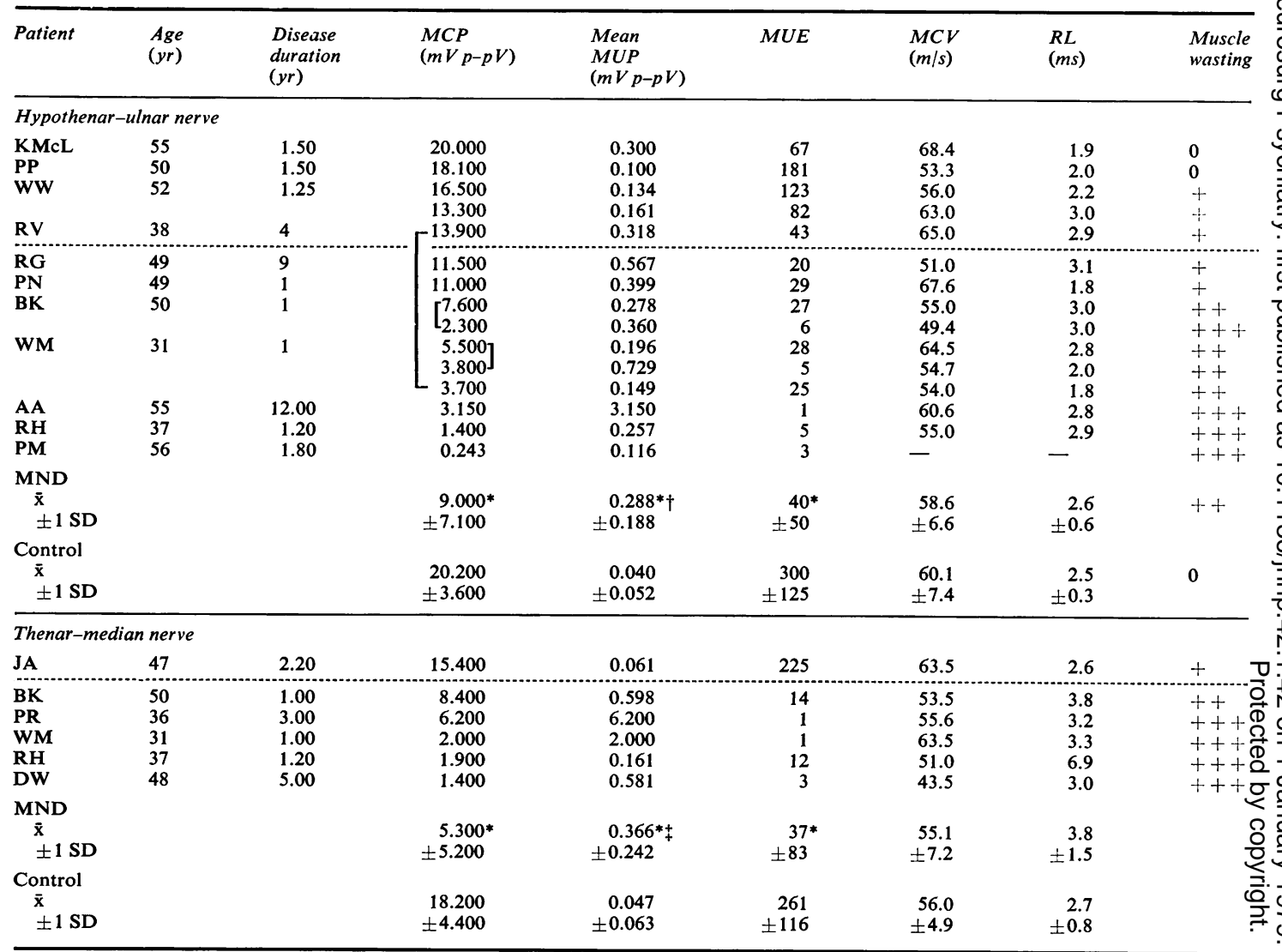

Vertical bars connect right and left nerves for the same subject.

Interrupted horizontal lines divide patient ht or $\mathrm{t}$ MCPs above or below the corresponding control 2 SD lower limit.

MCP=Maximum compound potential.

MUE $=$ Motor unit estimate.

$\mathrm{MCV}=$ Maximum conduction velocity in metres per second.

$\mathbf{R L}=\mathbf{R e s i d u a l}$ latency in milliseconds.

Muscle wasting. 0 none, + mild, ++ moderate,++ severe.

*Statistically significantly different from controls $\mathbf{P}<0.01$.

†Single motor units not included.

¥Examples where only one motor unit remained excluded from this calculation.

comparison of the relative numbers of low to high surface voltage MUPs. Moreover, even though motor unit force cannot be measured by these methods there is evidence for a significant relationship of surface voltage latency and tension (MilnerBrown and Stein, 1975; Kadrie et al., 1976; Brown, unpublished). In control subjects the relative percentages of small, medium, and large hypothenar MUPs recruited by threshold stimulation were 60,25 , and $15 \%$ respectively.

In MND, the distribution of MUP surface voltages was dramatically changed such that there was an increase (three to four times) in the relative contribution from large MUPs; only one-third the relative numbers of small MUPs were present (Fig. 2). Motor unit potential surface voltages over $1.0 \mathrm{mV}$, not present in controls, contributed up to $20 \%$ of the surviving MUP population in group 2 . Even to the late stages in MND, however, the lower surface voltage MUPs were present.

CHANGES IN RESPONSE TO REPETITIVE STIMULATION (TABLE 2)

Maximum compound potential

In control subjects increments are observed in the surface voltage of the fifth compared to the first maximum compound potential evoked by stimuli delivered at $1.0,0.3$, and 0.1 second intervals
ב ర 

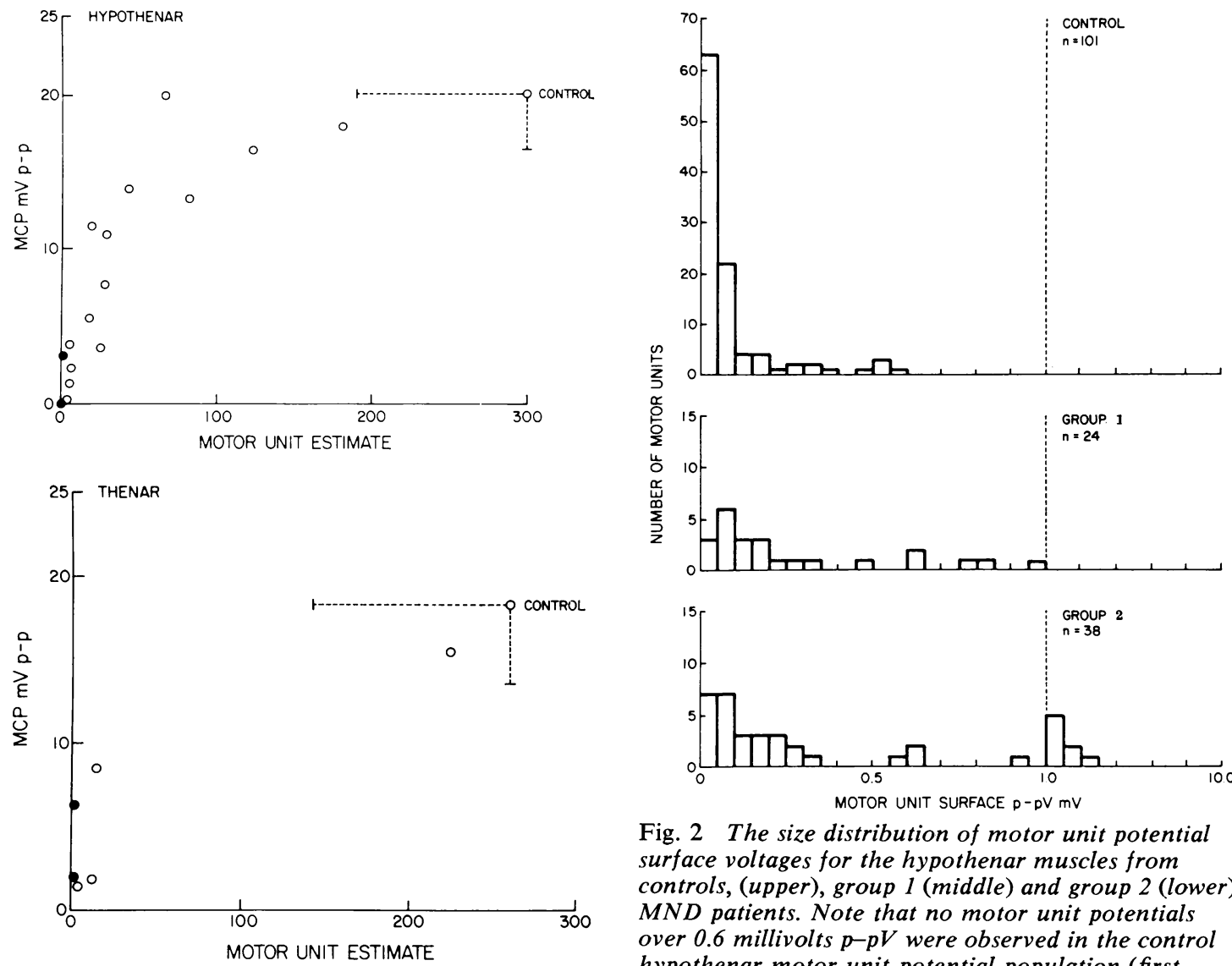

Fig. 1 Plot of the hypothenar (upper) and thenar (lower) maximum compound potential (MCP) surface peak-to-peak voltages $(p-p V)$ in millivolts $(m V)$ against the corresponding motor unit estimates (MUE). The solid circles represent single remaining motor units, open circles represent cases where more than one motor unit potential was present. The control mean and $-1 S D$ for the MCP $p-p V$ and the MUEs are illustrated.

(Kadrie and Brown, 1978a). In group 1 the increments in the maximum compound potential surface voltage were, by comparison, less but the differences compared to control subjects were significant only at 1.0 second stimulus intervals. In group 2, compared to control subjects, decrements in the maximum compound potential surface voltage were observed at 1.0 second stimulus intervals and even at 0.1 second stimulus intervals, little or no increment was observed. Overall, in MND decrement in the maximum compound potential voltage was never more than $10 \%$.

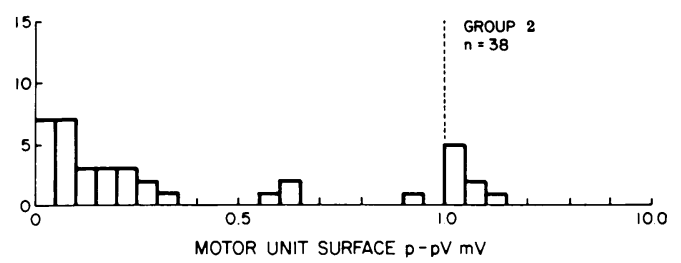

Fig. 2 The size distribution of motor unit potential surface voltages for the hypothenar muscles from controls, (upper), group 1 (middle) and group 2 (lower) MND patients. Note that no motor unit potentials over 0.6 millivolts $p-p V$ were observed in the control hypothenar motor unit potential population (first motor unit potentials recruited only). Note the change in scale factor indicated by the interrupted vertical line. Mean motor unit potential surface voltage: hypothenar control $=0.185 \mathrm{mV} p-p V \pm 0.2351 \mathrm{SD}$; hypothenar group $1 \mathrm{MND}=0.264 \mathrm{mV} p-p V \pm 0.286$ $1 S D$ (not significantly different from controls); hypothenar group $2 M N D=0.508 \mathrm{mV} p-p V \pm 0.771$ I SD $(P<0.01$ compared with controls and group 1$)$.

Table 2 Changes in the maximum compound potentials in response to repetitive stimulation

\begin{tabular}{lccc}
\hline & \multicolumn{2}{l}{$\begin{array}{l}\% \text { change } p-p \text { Vof fifth compared to first } \\
\text { MCP evoked by stimuli delivered at stimulus } \\
\text { intervals of }\end{array}$} \\
\cline { 2 - 4 } & $1.0 \mathrm{~s}$ & $0.3 \mathrm{~s}$ & $0.1 \mathrm{~s}$ \\
\hline $\begin{array}{l}\text { Control-hypothenar } \\
(\mathrm{n}=12)\end{array}$ & $2.9 \pm 2.0$ & $4.0 \pm 4.1$ & $13.5 \pm 9.3$ \\
$\begin{array}{l}\mathrm{MND} \text {-hypothenar } \\
\text { MCP }>13.0 \mathrm{mV} \\
(\mathrm{n}=5)\end{array}$ & $0.7 \pm 2.7^{*}$ & $1.6 \pm 1.2$ & $10.0 \pm 13.0$ \\
$\begin{array}{l}\mathrm{MCP}<13.0 \mathrm{mV} \\
(\mathrm{n}=10)\end{array}$ & $-1.3 \pm 6.5^{*}$ & $-1.1 \pm 10.3$ & $1.5 \pm 7.2^{*}$ \\
\hline
\end{tabular}

Values chosen include $\pm 1 \mathrm{SD}$.

*Statistically significant compared to controls $\mathrm{P}<0.05$. Abbreviations as in Table 1. 
Single motor units

For healthy hypothenar motor units the maximum decrement in the surface voltage of the fifth compared to the first MUP evoked by stimuli at 0.3 second intervals was $7 \%$. Therefore, for the purpose of this investigation, decrements were considered abnormal only if they exceeded $10 \%$. In MND group 1 patients, only one out of $24(4 \%)$ MUPs tested had a decrement that exceeded $10 \%$ (Fig. 3b) compared to a much higher proportion, 12 out of $38(32 \%)$, of group 2 MUPs tested. It is important, moreover, to note that the surface voltage decrements were much larger and more frequent for MUPs at the low end of the surface voltage range of the MUPs.

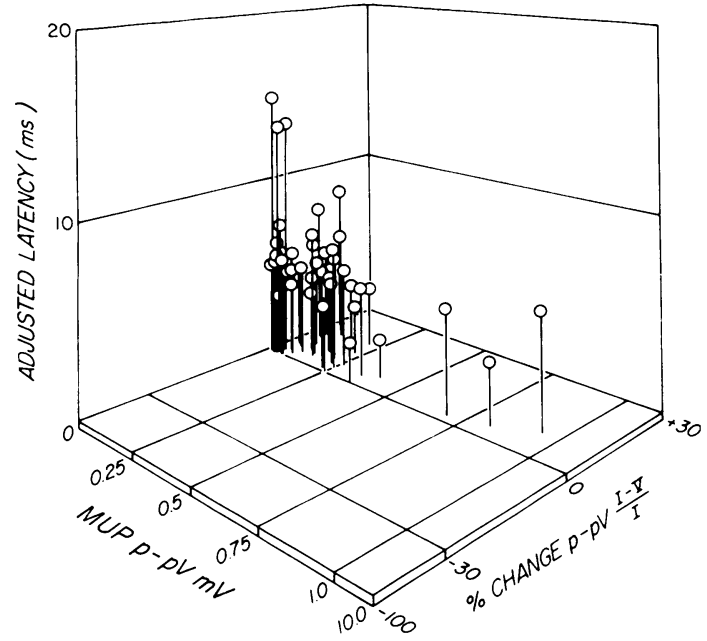

(a)

Fig. 3 The above plots illustrate the inter-relationships between motor unit potential surface peak-to-peak voltage $(p-p V)$, change in surface $p-p V$ evoked by stimulus trains at 0.3 second intervals, and the adjusted latencies for (a) the hypothenar control motor unit potentials (b) group 1 motor unit potentials, and (c) group 2 motor unit potentials. Note the change in scale factors at 1.0 millivolts for the surface $p-p V$ and at $30 \%$ for changes in the surface voltage evoked by stimulus trains. Mean changes in motor unit potentials $p-p V$ evoked by stimulus trains at 0.3 second intervals: control $=5.1 \% \pm 5.01 \mathrm{SD} ;$ group $1=0.7 \% \pm 6.81 \mathrm{SD}$ $(P<0.01$ compared with controls $) ;$ group $2=7.1 \% \pm$ $11.21 S D(P<0.01$ compared with group 1). Motor unit potential adjusted latencies: control $=5.3 \mathrm{~ms} \pm$ $2.61 \mathrm{SD} ;$ group $1=5.9 \mathrm{~ms} \pm 0.91 \mathrm{SD}$ (not significantly different from controls); group $2=7.3 \mathrm{~ms} \pm 1.51 \mathrm{SD}$ $(P<0.01$ compared with controls).
For example, only two out of $16(31 \%)$ of MUPs over $0.250 \mathrm{mV}$ had surface voltage decrements over $10 \%$, the mean reduction being $2.4 \%$. However, for MUPs less than $0.250 \mathrm{mV} \mathrm{p}-\mathrm{pV}$ the mean decrement was significantly larger $(10.4 \%)$, and a much higher proportion, 10 out of $22(45 \%)$, had decrements over $10 \%$. Hence at the later stages of MND the percentage of MUPs with abnormal decrements in surface voltage in response to repetitive stimuli was larger and the extent of the decrements much more abnormal. The decrements in surface voltage for individual MUPs were frequently much larger than for the corresponding maximum compound potentials evoked by supramaximal stimuli. Observations in the more limited

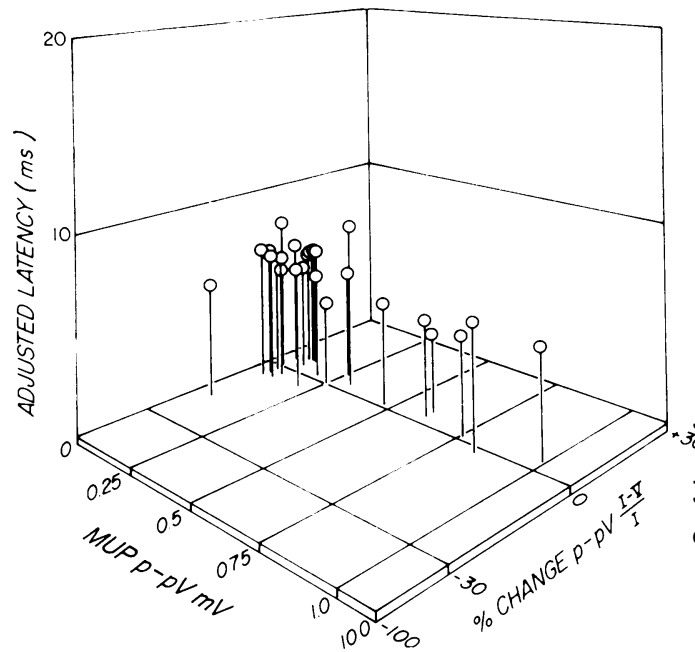

(b)

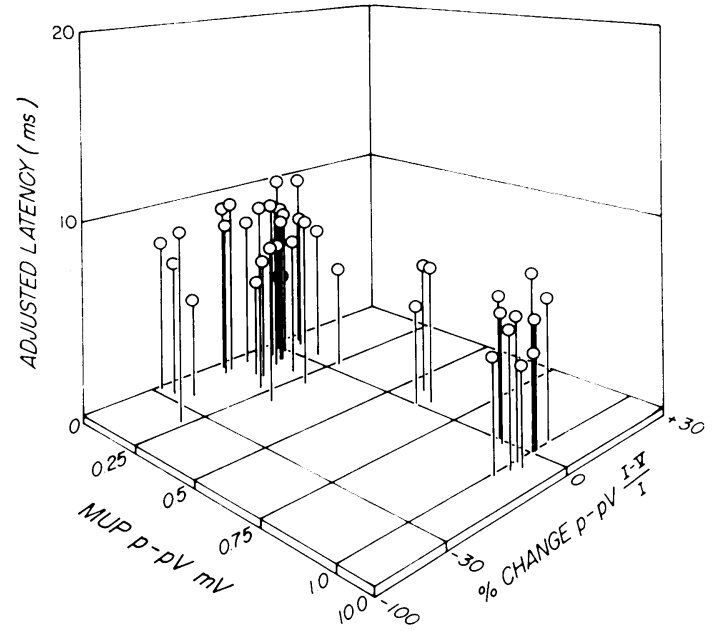

(c) 
numbers of control and MND thenar motor units tested were the same.

RELATIONSHIP BETWEEN MOTOR UNIT POTENTIAL SURFACE VOLTAGE, ADJUSTED LATENCY, RESPONSES TO REPETITIVE STIMULATION, AND THE RESPECTIVE MAXIMUM COMPOUND POTENTIAL VOLTAGE

To help illustrate the inter-relationships of MUP adjusted latency, surface voltage, and changes in the latter in response to stimulus trains, threedimensional plots have been constructed (Fig. 3). The control hypothenar motor unit pool, isolated by the multiple point stimulation method, was characterised by relatively large numbers of low surface voltage MUPs, rarity of decrements in the surface voltage in response to repetitive stimuli, and a relation between surface voltage and adjusted latency such that the longest adjusted latency MUPs had the lowest surface voltages, larger surface voltage MUPs having shorter latencies.

In MND group 1 patients, no significant change in the mean adjusted latency, compared to control subjects was observed but unlike the latter a relationship between MUP surface voltage and adjusted latency and the very long latency MUPs was not observed. In the more advanced group 2 patients, motor unit losses were much more severe (motor unit estimates less than 30 ) and significant $(P<0.01)$ increases in the mean MUP surface voltage and adjusted latency were observed. Decrements in MUP surface voltage at stimulus intervals of 0.3 seconds were also more abnormal and frequent in group 2 .

\section{Single surviving motor units}

The changes in the MUP surface voltage, residual latency, conduction velocity, and response to stimulus trains in the last surviving motor units in MND subjects are listed in Table 3. At these ter- minal stages three of the four MUPs were much larger in surface voltage $(2.0,6.2,3.2 \mathrm{mV})$ than in any control MUPs. Furthermore, the MUP surface voltage did not correlate with the corresponding MUP conduction velocity; the highest conduction velocity MUPs actually had the lowest surface voltages. Three of the four MUP conduction velocities were within one standard deviation of the control mean for the maximum motor conduction velocity $(60 \mathrm{~m} / \mathrm{s})$.

In two of the four MUPs, repeat testing revealed reductions in conduction velocity, larger decrements in surface voltage, an increase in residual latency and, in one of the two MUPs, a 53\% reduction in the MUP surface voltage (Fig. 4a). Interestingly, however, one MUP over a five month interval increased in surface voltage and conduction velocity, losing at the same time a previously evident abnormal decrement (Fig. 4b). The latter changes suggested possible recovery in this motor unit which was recorded from the patient with the longest MND duration (12 years). In one of the four MUPs no significant change in the measurement parameters was observed except for a reduction in residual latency.

\section{Discussion}

In motor neurone disease, motor unit loss, increases in the voltage of surviving MUPs, normal or close to normal maximum conduction velocities, and normal sensory fibre function are characteristic though not specific (Erminio et al., 1959; Mulder et al., 1959; Willison, 1962; Lambert, 1969; McComas et al., 1971; Hausmanowa-Petrusewicz and Kopec, 1973; Brown and Jaatoul, 1974; MilnerBrown et al., 1974; Norris, 1975; Stalberg et al., 1975). Our observations agreed with the above but provided new information on the relationship of the muscle maximum compound potential to motor

Table 3 Changes in the functional properties of the last surviving motor units

\begin{tabular}{|c|c|c|c|c|c|c|c|}
\hline \multirow[t]{2}{*}{ Patient } & \multirow[t]{2}{*}{$\begin{array}{l}\text { Time } \\
\text { interval } \\
\text { (months) }\end{array}$} & \multirow[t]{2}{*}{$\begin{array}{l}\text { Motor unit } \\
\text { potential } \\
(p-p V m V)\end{array}$} & \multicolumn{3}{|c|}{$\begin{array}{l}\% \text { change of } p-p V \text { of fifth compared to the } \\
\text { first MUP evoked by stimuli delivered at } \\
\text { stimulus intervals of }\end{array}$} & \multirow[t]{2}{*}{$\begin{array}{l}R L \\
(m s)\end{array}$} & \multirow[t]{2}{*}{$\begin{array}{l}C V \\
(\mathrm{~m} / \mathrm{s})\end{array}$} \\
\hline & & & $1.0 \mathrm{~s}$ & $0.3 \mathrm{~s}$ & $0.1 \mathrm{~s}$ & & \\
\hline WM & $\begin{array}{l}0 \\
4 \\
5\end{array}$ & $\begin{array}{l}2.000 \\
2.420 \\
3.250\end{array}$ & $\begin{array}{l}-1.2 \\
-14.7 \\
-9.8\end{array}$ & $\begin{array}{l}-12.5 \\
-17.5 \\
-12.6\end{array}$ & $\begin{array}{r}-15.9 \\
-7.4 \\
-\quad 5.6\end{array}$ & $\begin{array}{l}3.2 \\
3.5 \\
3.8\end{array}$ & $\begin{array}{l}63.5 \\
60.2 \\
76.9\end{array}$ \\
\hline $\begin{array}{l}\text { MUP_Fig. 4a } \\
\text { MUP_Fig. 4b }\end{array}$ & $\begin{array}{l}0 \\
5 \\
0 \\
5\end{array}$ & $\begin{array}{l}6.200 \\
1.300 \\
0.114 \\
0.253\end{array}$ & $\begin{array}{r}-4.4 \\
-8.2 \\
-18.1 \\
-13.8\end{array}$ & $\begin{array}{r}-7.9 \\
-36.0 \\
-19.2 \\
2.1\end{array}$ & $\begin{array}{r}-4.1 \\
-6.3 \\
-\quad 6.9\end{array}$ & $\begin{array}{l}3.2 \\
3.3 \\
3.9 \\
4.0\end{array}$ & $\begin{array}{l}55.6 \\
47.1 \\
68.3 \\
76.2\end{array}$ \\
\hline $\mathbf{A A}$ & $\begin{array}{l}0 \\
2\end{array}$ & $\begin{array}{l}3.150 \\
3.750\end{array}$ & $\begin{array}{l}0.9 \\
2.2\end{array}$ & $\begin{array}{l}3.5 \\
8.9\end{array}$ & $\begin{array}{r}8.8 \\
30.8\end{array}$ & $\begin{array}{l}2.8 \\
3.4\end{array}$ & $\begin{array}{l}58.4 \\
56.7\end{array}$ \\
\hline
\end{tabular}

Abbreviations as in Table 1. 


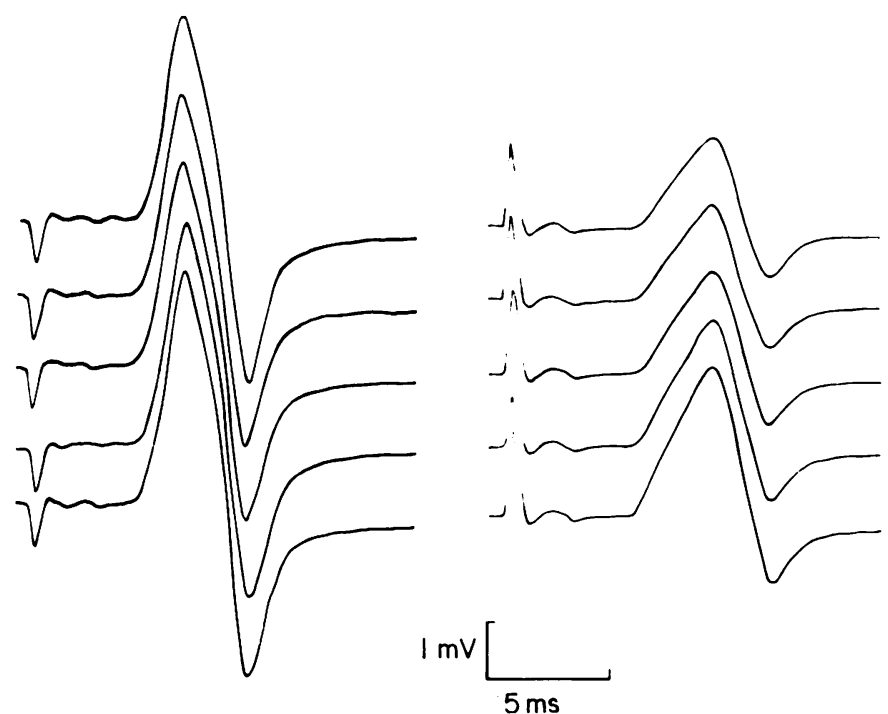

(a)

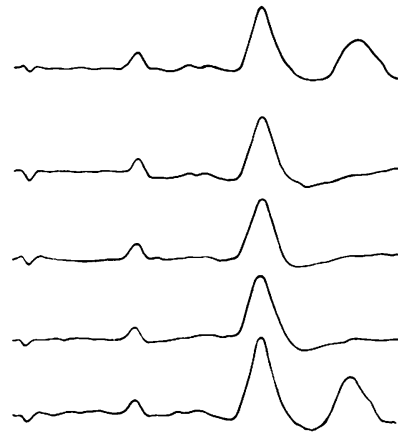

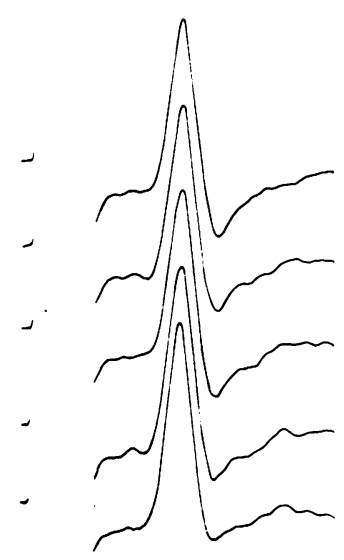

Fig. 4 Examples of changes in single remaining motor unit potentials in response to repetitive stimulation. Successively evoked motor unit potentials in response to stimuli delivered at 0.3 second intervals begin at the bottom. (a) This motor unit potential represents the largest of the four single surviving motor unit potentials. At the first test, (left) a $7.9 \%$ decrement in the surface $p-p V$ was observed. At the second test, (right) six to seven months later, not only was the $p-p V$ decrement larger $(36 \%)$ but the surface $p-p V$ of the motor unit potential was only $47 \%$ of that at the first examination. (b) This motor unit potential, at the first test, (left), had a surface $p-p V$ decrement of over $19 \%$. Repetitive motor unit potential discharges in response to single stimuli delivered to the nerve were observed at the same examination. Five months later (right), not only was the surface $p-p V$ over two times larger and the conduction velocity increased but the $p-p V$ decremen was no longer evident. Note the antidromic sensory nerve action potential preceding the motor unit potential, most obviously at the time of the first examination.

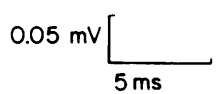

(b) unit numbers, the distribution of MUP voltages, and the relationship between MUP surface voltage and the changes in MUP surface voltage evoked by trains of stimuli. The observations lead to new questions for other investigations.

The maximum compound potential surface voltage is likely to be closely related to the total number of active muscle fibres present, though other factors including muscle fibre diameter, the degree of synchronisation in the summation of muscle fibre action potentials, and the general distribution and distance of the muscle fibre population from the surface recording electrode are also important factors. It is not unreasonable to infer that maintenance of a normal or near normal maximum compound potential voltage probably means that surviving motor units have reinnervation capacities sufficient to keep up with the rate of muscle fibre denervation. Only when the reinnervation capacity falls below the denervation rate, as when too few numbers of healthy motor units remain, the distances required for axonal sprouting, or connective tissue barriers force a limit to the reinnervation, would reduction 
in the maximum compound potential voltage be expected.

Our observations on the changes in the relationship between the surface voltage of the maximum compound potential and the corresponding motor unit estimates that parallel the advance of MND supported the above speculations. Further support came from earlier evidence that muscle strength, judged clinically, or the maximum twitch tension could be maintained in the normal range by as few as $10-20 \%$ of the normal estimated number of motor units (McComas et al., 1971; Brown, 1973). Hence, in MND, it was only when motor unit losses became severe (less than one-third of the control lower limit) that the surface voltages for the muscle maximum compound potential were observed to fall below the two standard deviation lower limit for control subjects.

Progression in MND was characterised by an increase in the average MUP surface voltage, relative increase in the numbers of larger surface voltage MUPs, and the recognition of MUPs much larger than the largest known control MUPs. These observations were the likely consequence of motor unit loss and the enlargement through reinnervation of the surviving motor units. Even at late stages in MND, however, the lower surface voltage MUPs were present. The latter low surface voltage MUPs could represent motor units that had somehow survived with little change in voltage, or they could represent the late stage degeneration of units with previously larger voltage MUPs.

The MUP voltage measured on the surface over the muscle is related to the motor unit force (Appleberg and Emonet-Denaud, 1967; Kernell et al., 1975; Milner-Brown and Stein, 1975; Kadrie et al., 1976). Unfortunately the methods in this investigation precluded proper measurement of the force of the motor units. In MND, however, the surface voltage may be a better indicator of the total number of muscle fibres innervated per motor unit force because electromechanical uncoupling could result in a lower force per motor unit than expected (Milner-Brown et al., 1974). In this investigation the largest MUP surface voltage was $6 \mathrm{mV}$, over fifty times larger than the average control MUP surface voltage and six times the largest surface voltage for a healthy MUP. The presence of electromechanical uncoupling was suggested by the observation that this very large voltage MUP was accompanied by twitch that was only just recognisable.

Abnormalities of neuromuscular transmission have been reported frequently in MND (Lambert and Mulder, 1957; Mulder et al., 1959; Simpson and Lenman, 1959; Simpson, 1966; Lambert, 1969;
Miglietta, 1971; Desmedt, 1973; Brown and Jaatoul, 1974; Stalberg et al., 1975; Schwartz et al., 1976). In this investigation decrements in the surface voltage of MUPs or the maximum compound potential were infrequent at early stages in MND, particularly if no muscle fibre wasting was evident or the maximum compound potential was above the control two standard deviation lower limit. Overall, surface voltage decrements were more common and abnormal in the lower surface voltage MUPs, and the decrements became particularly frequent and severe at the later stages in MND. Even the larger surface voltage MUPs had decrements in response to trains of stimuli at late stages in MND.

What factors could account for the decrements in voltage evoked by nerve stimulation? Synaptic transmission failure at neuromuscular junctions undergoing degeneration was probably the single most important factor. Neuromuscular transmission failures at immature regeneration junctions were an unlikely explanation for the much larger voltage decrements observed at the later stages in MND. Impulse block at axon branches or axon terminals was unlikely to explain voltage decrements at stimulus intervals as long as 0.3 or 1.0 seconds (Desmedt, 1973).

The degree of synchronisation in the summation of muscle fibre action potentials was an important factor to consider. Theoretically, such synchronisation should be increased in conditions where the chance for interaction between muscle fibres was higher (Katz and Schmitt, 1940), for example where fibre grouping occurs as the consequence of reinnervation. More synchronisation has not, however, been observed in MUPs after peripheral nerve section and resuture (Brown, unpublished). Therefore, the most important factor in determining the decrements in surface voltage for MUPs as well as the maximum compound potential was probably abnormal synaptic transmission at neuromuscular junctions developing degenerative changes.

The observation that MUPs at the lower end of the surface voltage range in MND had the largest voltage decrements could mean that their motor units were attacked selectively in this disease. There is no evidence for preferential abnormalities in one or other type of motor unit in MND (Dubowitz and Brooke, 1973). The MND observations, together with the previous evidence on myasthenia gravis (Kadrie and Brown, 1978b), prompted us, instead, to suggest the hypothesis that the margin for safe neuromuscular transmission was normally less for motor units with lower rather than larger surface voltage MUPs 
(Kadrie and Brown, 1978b). This hypothesis has received a measure of experimental support (Gertler and Robbins, 1978).

The observation that surface voltage decrements were less for the maximum compound potential than for individual MUPs, particularly the lower surface voltage units, was probably the result of the biased selection of the latter MUPs by the threshold or near threshold motor nerve stimuli (Kadrie et al., 1976; Kadrie and Brown, 1978b). Thus the maximum compound potential represents the summation of larger surface voltage MUPs that have relatively less voltage decrements and larger decremental responses from lower surface voltage MUPs.

There was evidence for a breakdown in the orderly relationship between MUP surface voltage and latency in MND. In control subjects there was an approximate relationship between MUP surface voltage and the corresponding adjusted latency (Freund et al., 1975; Kadrie et al., 1976; Kadrie and Brown, 1978a) such that the lowest surface voltage MUPs have the longest latencies. In MND, no correlation between MUP surface voltage and adjusted latency was observed despite significant increases in the average MUP adjusted latency at later stages. Contrary to what could happen theoretically if larger demands were placed on the motoneurone by reinnervation resulting in an increase in the axon diameter, the largest MUPs (over $2 \mathrm{mV}$ ) did not have very high conduction velocities. Conversely, lower conduction velocities were not necessarily observed in the lower surface voltage MUPs. There were, for example, low surface voltage MUPs with quite high conduction velocities and very large surface voltage MUPs with normal or lower than normal conduction velocities.

At any stage in MND, the motor unit population must be quite complex. Firstly, superimposed on the normal range for MUP surface voltage and latency are motor unit losses, presumably involving all motor units irrespective of the functional type of unit. Secondly, denervation and consequent reinnervation result in abnormal increases in the numbers of muscle fibres innervated per motor unit, this in part accounting for the larger surface voltage of the MUPs. Thirdly, further advances in the disease probably result in dysfunctional changes and death of motor units that by reinnervation had supported larger than normal numbers of muscle fibres. Especially at later stages, therefore, there is probably a mixture of healthy normal, healthy but enlarged motor units, and dysfunctional motor units undergoing degeneration. It is also impossible to tell the previous history of a motor unit from the surface voltage or latency of its MUP. Abnormal decrements in surface voltage evoked by repetitive stimuli, however, probably indicate degenerative changes in that motor unit.

One aspect requiring further investigation was that of single surviving motor units in MND. The MUPs of two such surviving units were observed to undergo changes, including reductions in surface voltage and conduction velocity accompanied by an increase in the residual latency that probably indicated progressive degenerative changes in those motor units. The fact that two such units were still present at the end of five months is itself remarkable in view of the extraordinarily rapid rates of loss of motor units calculated for the early stages of MND (Brown and Jaatoul, 1974). Most interesting was the single MUP that underwent an increase in surface voltage and conduction velocity, changes that suggested a possible recovery. Perhaps in certain cases of MND there are motor unit survivors, particularly in patients having a longer than usual history as in this example (12 years).

\section{References}

Appelberg, B., and Emonet-Denaud, F. (1967). Motor units of the first superficial lumbrical muscle of the cat. Journal of Neurophysiology, 30, 154-160.

Brown, W. F. (1972). A method for estimating the number of motor units in thenar muscles and the changes in motor unit counting with aging. Journal of Neurology, Neurosurgery, and Psychiatry, 35, 845-852.

Brown, W. F. (1973). Functional compensation of human motor units in health and disease. Journal of the Neurological Sciences, 20, 199-209.

Brown, W. F., and Jaatoul, N. (1974). Amyotrophic lateral sclerosis. Archives of Neurology (Chicago), 30, 240-248.

Campbell, M. J., McComas, A. J., and Petito, F. (1973). Physiological changes in aging muscles. Journal of Neurology, Neurosurgery, and Psychiatry, 36, 174-182.

Desmedt, J. E. (1973). The neuromuscular disorder in myasthenia gravis. I. Electrical and mechnical response to nerve stimulation in hand muscles. II. Pre-synaptic cholinergic metabolism, myasthenic-like syndromes and a hypothesis. In New Developments in Electromyography and Clinical Neurophysiology, Vol. 1, pp. 241-342. Edited by J. E. Desmedt. Karger: Basel.

Dubowitz, V., and Brooke, M. H. (1973). Muscle Biopsy: A Modern Approach, Vol. 2 in the Major Problems in Neurology. Edited by J. N. Walton. W. B. Saunders: London.

Erminio, F., Buchthal, F., and Rosenfalck, P. (1959). Motor unit territory and muscle fiber concentration in paresis due to peripheral nerve injury and 
anterior horn cell involvement. Journal of Neurology, 9, 659-671.

Freund, H. J., Budingen, H. J., and Dietz, V. (1975). Activity of single motor units from human forearm muscles during voluntary isometric contractions. Journal of Neurophysiology, 38, 933-946.

Gertler, R. A., and Robbins, N. (1978). Differences in neuromuscular transmission in red and white muscles. Brain Research, 142, 160-164.

Hausmanowa-Petrusewicz, I., and Kopec, J. (1973). Motor nerve conduction velocity in anterior horn lesions. In New Developments in Electromyography and Clinical Neurophysiology, Vol. 2, pp. 298-305. Edited by J. E. Desmedt. Karger: Basel.

Kadrie, H. A., and Brown, W. F. (1978a). Neuromuscular transmission in human single motor units. Journal of Neurology, Neurosurgery, and Psychiatry, 41, 193-204.

Kadrie, H. A., and Brown, W. F. (1978b). Neuromuscular transmission in myasthenic single motor units. Journal of Neurology, Neurosurgery, and Psychiatry, 41, 205-214.

Kadrie, H. A., Yates, S. K., Milner-Brown, H. S., and Brown, W. F. (1976). Multiple point electrical stimulation of ulnar and median nerves. Journal of Neurology, Neurosurgery, and Psychiatry, 39, 973985.

Kernell, D., Ducati, A., and Sjoholm, H. (1975). Properties of motor units in the first deep lumbrical muscle of the cat's foot. Brain Research, 98, 37-55.

Katz, B., and Schmitt, O. H. (1940). Electric interaction between two adjacent nerve fibres. Journal of Physiology, 97, 471-488.

Lambert, E. H. (1969). Electromyography in amyotrophic lateral sclerosis. In Motor Neuron Diseases, pp. 135-153. Edited by F. H. Norris and L. T. Kurland. Grune and Stratton: New York.

Lambert, E. H., and Mulder, D. W. (1957). Electromyographic studies in amyotrophic lateral sclerosis. Proceedings of Staff Meetings of the Mayo Clinic, 32, 441-446.

McComas, A. J. (1977). Neuromuscular Function and Disorders. Butterworths: London.

McComas, A. J., Sica, R. E. P., Campbell, M. J., and Upton, A. R. M. (1971). Functional compensation in partially denervated muscles. Journal of Neurology, Neurosurgery, and Psychiatry, 34, 453-460.
Miglietta, O. E. (1971). Myasthenic-like response in patients with neuropathy. American Journal of Physical Medicine, 50, 1-16.

Milner-Brown, H. S., and Brown, W. F. (1976). New methods of estimating the number of motor units in a muscle. Journal of Neurology, Neurosurgery, and Psychiatry, 39, 258-265.

Milner-Brown, H. S., and Stein, R. B. (1975). The relation between the surface electromyogram and muscular force. Journal of Physiology, 246, 549569.

Milner-Brown, H. S., Stein, R. B., and Lee, R. G. (1974). Contractile and electrical properties of human motor units in neuropathies and motor neurone disease. Journal of Neurology, Neurcsurgery, and Psychiatry, 37, 670-676.

Mulder, D. W., and Howard, F. M. (1976). Patient resistance and prognosis in amyotrophic lateral sclerosis. Mayo Clinic Proceedings, 51, 537-541.

Mulder, D. W., Lambert, E. H., and Eaton, L. M. (1959). Myasthenic syndrome in patients with amyotrophic lateral sclerosis. Neurology (Minneapolis), 9, 627-631.

Norris, F. H. (1975). Adult spinal motor neuron disease. In Handbook of Clinical Neurology, Vol. 22, Part II. Edited by P. J. Vinken and G. W. Bruyn. North-Holland: Amsterdam.

Schwartz, M. S., Stalberg, E., Schiller, H. H., and Thiele, B. (1976). The reinnervated motor unit in man. Journal of the Neurological Sciences, 27, 303-312.

Simpson, J. A. (1966). Disorders of neuromuscular transmission. Proceedings of the Royal Society of Medicine, 59, 993-997.

Simpson, J. A., and Lenman, J. A. R. (1959). The effect of frequency of stimulation in neuromuscular disease. Electroencephalography and Clinical Neurophysiology, 11, 604.

Stalberg, E., Schwartz, M. S., and Trontelj, J. V. (1975). Single fibre electromyography in various processes affecting the anterior horn cell. Journal of the Neurological Sciences, 24, 403-415.

Willison, R. G. (1962). Discussion: motor neurone disease. Proceedings of the Royal Society of Medicine, 55, 44-48. 